\title{
Athérosclérose et hyperhomocystéinémie : un facteur de risque génétique à prendre désormais en compte
}

Dans l'homocystinurie, maladie récessive autosomique connue depuis plusieurs décennies, la relation entre tableau clinique et signes biologiques fut rapidement établie: les thromboses et les nombreux accidents athéromateux précoces étaient liés aux concentrations très élevées d'homocystéine et de méthionine dans le sang des malades. Une déficience homozygote en cystathionine $\beta$ synthase (CBS), enzyme qui catalyse la formation de cystathionine à partir d'homocystéine et de sérine est la cause de cette grave maladie métabolique.

La notion de la toxicité de l'hyperhomocystéinémie pour les parois endothéliales n'est donc pas nouvelle mais pendant longtemps elle ne parut pas présenter d'intérêt pour les cardiologues dans leurs recherches sur les facteurs de risque des maladies coronaires et de l'athéromatose. En effet, l'homocystinurie par déficit en CBS est une maladie rare, cliniquement caractéristique, et les études réalisées chez les hétérozygotes ne montrèrent aucune augmentation significative des accidents cardiovasculaires. En outre, des mesures de l'activité de la CBS effectuées dans des groupes de patients atteints d'athéromatose précoce ne mirent en évidence aucune diminution de l'activité enzymatique. Par la suite, quand le gène codant pour cette enzyme fut cloné et les mutations répertoriées chez des malades atteints d'homocystinurie et leurs parents, aucune augmentation de fréquence significative de ces mutations, à l'état hétérozygote, ne fut observée dans les groupes de coronariens ou d'athéromateux étudiés [1].
D'autres enzymes interviennent aussi dans le métabolisme de l'homocystéine (figure 1). La méthylène tétrahydrofolate réductase (MTHFR), une flavoprotéine cytosolique, agit avec le dinucléotide adénine flavine comme cofacteur en transformant la 5-10 méthylène tétrahydrofolate en 5méthyl tétrahydrofolate, indispensable à la reméthylation de l'homocystéine en méthionine. Mais, là encore, la déficience homozygote de la MTHFR a une expression phénotypique particulière avec un large éventail de manifestations cliniques, allant de la mort périnatale à des troubles moteurs sévères, avec crises d'épilepsie et manifestations psychiatriques ultérieures [2]. Les anomalies vasculaires y sont présentes mais ne dominent pas le tableau.

Restait donc cette hyperhomocystéinémie modérée, fréquemment observée chez les athéromateux, qui pouvait dépendre aussi bien de facteurs génétiques que de facteurs nutritionnels [3]. Nous allons voir qu'il s'agit en fait d'un très joli modèle d'écogénétique.

Une première indication fut la découverte d'un variant thermolabile de la MHTFR, dont l'activité spécifique était réduite de $50 \%$ par rapport aux témoins, avec $30 \%$ d'activité résiduelle (qui est normalement de $50 \%$ ) après chauffage à $46^{\circ} \mathrm{C}$ pendant cinq minutes. Responsable d'une hyperhomocystéinémie modérée, elle fut retrouvée avec une fréquence significative chez les malades vasculaires et les coronariens, en particulier ceux qui avaient justement une hyperhomocystéinémie [4]. Aussi, dès que le gène codant pour la MTHFR, localisé en $1 p 36.3$, [5,6] fut cloné, la mutation ayant pour conséquence la production d'une enzyme thermolabile à activité spécifique diminuée fut recherchée. Il s'agit d'une transition $\mathrm{C} \rightarrow \mathrm{T}$ en position 677, qui introduit un site de restriction pour HinfI. Comme l'a montré l'étude de l'expression du gène in vitro par Escherichia coli, elle a effectivement pour conséquence de rendre thermolabile la protéine en substituant une valine à une alanine dans une région conservée de cette enzyme dans toute les espèces animales. Chez l'homme, la fréquence de ce variant est de 0,38 . Il n'est cependant pas réparti de façon homogène [7]: répandu chez les Canadiens français (comme les mutations du récepteur des LDL), dans certains pays européens, au Moyen-Orient, et au Japon où l'on compte environ $15 \%$ d'homozygotes, il est plus rare en Finlande $(5,4 \%)$ et au Danemark $(5,2 \%)$ et probablement encore plus rare en Afrique [8]. Comme viennent de le confirmer deux études récentes $[9,10]$, sa fréquence est significativement plus élevée chez les coronariens et les vasculaires. Dans une population danoise, en excluant les autres facteurs de risque (hypertension, diabète, hyperlipoprotéinémie), la prévalence des homozygotes dans les groupes d'accidents vasculaires précoces (AVP) est de $15 \%$ alors qu'elle n'est que de $5,2 \%$ chez les témoins. Inversement, dans ces groupes d'AVP, les sujets homozygotes pour $677 \mathrm{C} \rightarrow \mathrm{T}$ sont bien ceux qui ont une hyperhomocystéinémie modérée. Enfin, si l'on sélectionne des sujets avec hypercystéinémie et AVP, on retrouve le variant thermolabile dans $28 \%$ des cas. 
On voit donc que la responsabilité de ce variant est loin d'être négligeable. Il agit par l'intermédiaire de l'hyperhomocystéinémie, dont on connaît encore mal le mécanisme d'action sur les parois endothéliales mais dont on sait déjà qu'elle peut être responsable de toutes sortes d'atteintes vasculaires: maladies coronaires, accidents vasculaires cérébraux et périphériques, et thromboses veineuses [11]. L'âge d'apparition des premiers symptômes s'étale de 18 à 50 ans.

Mais les études biochimiques en cours nous apportent encore des précisions supplémentaires: parmi les homozygotes pour ce variant $M T H$ $F R$, ce sont surtout les sujets carencés en acide folique qui présentent une hyperhomocystéinémie $[12,13]$. Si l'homocystéinémie est deux fois plus élevée à jeun chez les sujets $(+/+)$ pour ce variant $677 \mathrm{C} \rightarrow \mathrm{T}$ que chez les sujets $(+/-)$ ou $(-/-)$, la prise quotidienne d'acide folique est très efficace pour la réduire [14, 15].

En reprenant les données d'une méta-analyse effectuée aux État-Unis [16], où la mortalité par cardiopathies ischémiques est d'environ 50 000/an, Arno Motuslky [8] a calculé que l'adjonction de $350 \mu \mathrm{g}$ d'acide folique pour $100 \mathrm{~g}$ d'aliment (comme des produits céréaliers par exemple) pourrait diminuer de $9 \%$ les décès chez l'homme et dans un pourcentage beaucoup plus élevé chez la femme, chez laquelle les autres facteurs de risque sont moins nombreux.

En France, la prescription de vitamines est diversement appréciée. Certes, il existe un consensus pour la prise orale quotidienne d'acide folique par les femmes enceintes pendant les quatre premiers mois de grossesse. Ce traitement diminue de plus de $50 \%$ la survenue de défauts de fermeture du tube neural chez l'enfant à naître. Mais un apport systématique n'a jamais été envisagé dans la population. Comme pour les défauts du récepteur des LDL et de l'apolipoprotéine B [17], le dosage de l'homocystéinémie devrait faire partie des tests biologiques de recherche des sujets à risque, avant toute analyse moléculaire. Enfin, en

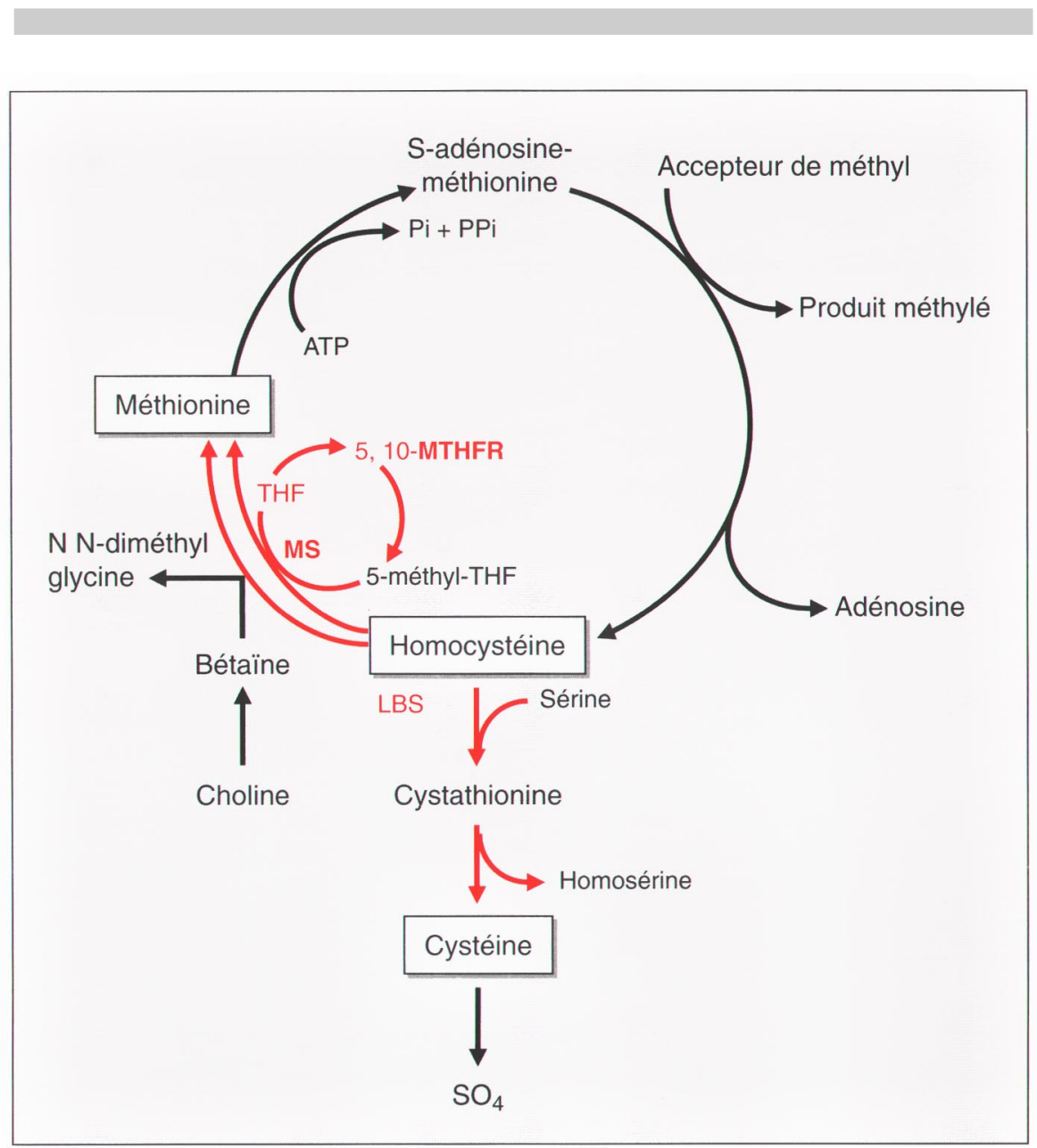

Figure 1. Métabolisme de I'homocystéine. On connaît des anomalies délétères dans deux voies du métabolisme de l'homocystéine: la voie de synthèse de la cystéine, dans laquelle l'enzyme cystathionine $\beta$ synthase (CBS) permet la formation de cystathionine à partir d'homocystéine et de sérine, et la voie de synthèse de la méthionine qui fait intervenir le 5-méthyl-tétrahydrofolate (THF) formé sous l'action de la 5,10-méthylène-tétrahydrofolate réductase (MTHFR). Le déficit en CBS est responsable de I'homocystinurie, maladie rare mettant les reins en péril; le déficit en MTHFR, en revanche, est fréquent, souvent associé à des troubles vasculaires, en particulier coronaires. MS : méthionine synthase.

taires permettant de mesurer le rôle exact $\mathrm{du}$ variant $677 \mathrm{C} \rightarrow \mathrm{T}$ dans la maladie athéromateuse, les cardiologues devraient dès maintenant ajouter aux recommandations d'un régime pauvre en acides gras saturés et en cholestérol celles d'une alimentation riche en acide folique, c'est-àdire en légumes verts et agrumes.

S.G.
1. Whitehead AS, Ward P, Tan S, Naughten E, Kraus JP, Sellar GC, McConnell DJ, et al. The molecular genetics of homocystinuria, hyperhomocysteinemia, and premature vascular disease in Ireland. In: Mato MJ, Caballero A, eds. Methionine metabolism: molecular mechanisms and clinical implications. Madrid: Bouncopy, 1994 : 79-83.

2. Rosenblatt DS. Inherited disorders of folate transport and metabolism. In: Scriver CR, Beaudet AL, Sly WS, Valle D, eds. The metabolic basis of inherited disease. 6th ed. New York: McGraw-Hill 1989: 2049-64. 
3. Clarke R Daly L, Robinson K, Naughten E, Canalane S, Fowler B, Graham I. Hyperhomocysteinemia: an independant risk factor for vascular disease. N Engl J Med 1991 ; 324: 1149-55.

4. Kang SS, Womg PWK, Susmano A, Sora J, Norusis $\mathrm{M}$, Ruggie N. Thermolabile methylene tetrahydrofolate reductase : an inherited risk factor for coronary artery disease. Am J Hum Genet 1991 ; 48 : 536-45.

5. Goyette P, Sumner IS, Milos R, Duneau AMV, Rosenblatt DS, Matthews RG, Rozen R. Human methylene tetrahydrofolate reductase: isolation of cDNA, mapping and mutation identification. Nature Genet 1994; 7 : 195-200.

6. Goyette P, Frosst P, Rosenblatt DS, Rozen R. Seven novel mutations in the methylene tetrahydrofolate reductase gene and genotype/phenotype correlation in severe methylene-tetrahydrofolate reductase deficiency. Am J Hum Genet 1995; 56 : 1052-9

7. Frosst P, Bloom HJ, Milos R, Goyette P, Sheppard CA, Mathews RG, Boers GHJ et al. A candidate genetic risk factor for vascular disease: a common mutation in methylene-tetrahydrofolate reductase. Nature Genet 1995; 10 : 111-3.

8. Motulsky A. Nutritional ecogenetics: homocysteine-related arteriosclerotic vascular disease, neural tube defects, and folic acid. Am J Hum Genet $1996 ; 58$ : 17-20.

9. Engbersen AM, Franken DG, Boers GHJ, Stevens EMB, Trijbels FJM, Blom HJ. Thermolabile 5,10-methylenetetrahydrofolate reductase as a cause of mild hyperhomocysteinemia. Am J Hum Genet 1996 ; 56 : 142-50.

10. Kluijtmans LA, Van den Henvel LPWJ, Boers GHJ, Frosst P, Stevens EMB, van Oost BA, den Heijer M, Trijbels FJM, Rozen R, Blom HJ. Molecular analysis in mild hyperhomocysteinemia: common mutation in the méthylène tétrahydrofolate reductase gene is a genetic risk factor for cardiovascular disease. Am J Hum Genet 1996; 58: 35-41.

11. Bloom HJ, van de Molen E. Pathobiochemical implications of hyperhomocysteinemia. Fibrinolysis 1994; 8 (suppl 2); 86-7.

12. Rozen R, Jacques P, Bostom A, Ellison C, Williams R, Rosenberg I, Selhub J. Interaction of a common mutation in methylenetetrahydrofola- te reductase (MHTFR) with low folate status in hyperhomocysteinemia. Am J Hum Genet 1995; suppl 57: A250.

13. Rosenblatt DS, Inherited disorders of folate transport and metabolism. In : Scriver CR, Baudet AL, Sly WS Valle D, eds. The metabolic and molecular bases of inherited diseaeses, 7th ed: New York: McGraw-Hill, 1995 : 3111-25.

14. Sehlub J, Jacques Pf, Wilson PWF, Rush D, Rosenberg IH. Vitamin status and intake as primary determinants of homocysteinemia in an ederly population. J Am Med Ass 1993; 270 : 2693-8.

15. Franken DG, Boers GHJ, Blom HJ, Trijbels JMF, Kloppenborg PWC. Treatment of mild hyperhomocysteinemia in vascular disease patients. Arterioscler Thromb 1994; 14: 465-70.

16. Boushey CJ, Beresford SSA, Omenn GS, Motulsky AG. A quantitative assessment of plasma homocysteine as a risk factor for arteriosclerotic vascular disease: profitable benefits of increasing folic acid intakes. J Am Med Ass 1995; 274: 1049-57.

17. Benlian P, Loux N. Hétérogénéité des mutations du récepteur LDL dans l'hypercholestérolémie familiale. médecine/sciences $1991 ; 10$ : 1052-60. 